\title{
The Effect of Metal Oxycarbonyl Group on the Claisen Rearrangement
}

\author{
Yutaka OKADA*, Takashi Ueno and Takatoshi HaYashi \\ Department of Applied Chemistry, Faculty of Science and Engineering, Ritsumeikan University \\ (1-1-1, Nojihigashi, Kusatsu-shi, 525-8577 JAPAN)
}

Edited by A. Masuyama, Osaka Univ., and accepted May 2, 2003 (received for review March 27, 2003)

\begin{abstract}
The Claisen rearrangement of 2-allyloxybenzoic acid and its alkali and alkalineearth metal salts was carried out. For the alkali metal salts, no accelerated effect was observed. There is also no accelerated effect for alkali metal salts in a nonpolar solvent. However, a significantly high reactivity was observed for the alkaline-earth metal salts in a nonpolar solvent. This is attributed to the chelate formation between the ethereal oxygen and the - $\mathrm{COO}^{-}$.

Key words: Claisen rearrangement, substituent effect, alkali metal, alkaline-earth metal, chelate effect
\end{abstract}

\section{Introduction}

The authors found an accelerated effect due to the ortho-carboxyl group for the Claisen rearrangement of 2-allyloxybenzoic acid (AOBA) (1). In this study, the alkali and alkaline-earth metal salts of AOBA were used for the rearrangement. For the alkali metal salts, no accelerated effects were noticed. However, the rearrangement of the alkaline-earth metal salts was significantly accelerated in a nonpolar solvent.

\section{Experimental}

\section{1 Preparation of Materials}

The preparation and physical properties of AOBA and 3-allyl-2-hydroxybenzoic acid were reported in a previous paper (1).

\section{$2 \cdot 2$ Measurements on the Claisen Rear- rangement of 2-allyloxybenzoic Acid (AOBA)}

The Claisen rearrangement was conducted in a 100 $\mathrm{mL}$ flask equipped with an air condenser under a nitro- gen atmosphere. AOBA and solvent were placed in the flask that was heated to a constant temperature in an oil bath. After a measured time, $0.5 \mathrm{~mL}$ of the reaction mixture was treated with $2 \mathrm{~mL}$ of methanol. When decalin was used as the reaction solvent, the solute was extracted by $1 \mathrm{~mL}$ of diethyleneglycol (DEG). The amounts of the unreacted material and products were determined by HPLC. The HPLC analysis was operated by Shimadzu LC-6A with UV detector SPD-6A. ODS column (Cosmosil 5C18 (nacalai tesque)) was used, and the elution was done by methanol.

\subsection{Measurements on the Claisen Rear- rangement of the Alkali or Alkaline- Earth Metal Salts of AOBA}

The Claisen rearrangement was completed similar to the free AOBA setup. After sampling $0.5 \mathrm{~mL}$ of the reaction mixture, $0.1 \mathrm{~N}-\mathrm{HCl}$ was added, and the solute was extracted by $2 \mathrm{~mL}$ of diethyl ether. The ether layer was separated followed by evaporation of the ether. The residue was then analyzed by HPLC.

\footnotetext{
* Correspondence to: Yutaka OKADA, Department of Applied Chemistry, Faculty of Science and Engineering, Ritsumeikan University, 1-1-1, Nojihigashi, Kusatsu-shi, 525-8577 JAPAN

E-mail: ygvictor@se.ritsumei.ac.jp
} 


\section{Results and Discussion}

As shown in Fig. 1, for the rearrangement of AOBA, a decarboxylate product $(\underline{B})$ forms together with the normal ortho-rearranged product (A) (2). AOBA in this study yielded these two products in all the solvents used: decalin, diethyleneglycol monoethylether (DEGMEE) and DEG. Its alkali and alkaline-earth metal salts also afforded the same products.

As an example, the rearrangement yield and the ratio of decarboxylation versus reaction time are shown in Fig. 2. Here the yield is defined as the total amounts of (A) and (B), and is equal to the conversion of the substrate. The ratio of decarboxylation is defined as the ratio of $(\mathrm{B})$ to $(\underline{A})+(\underline{B})$. The ratio was essentially constant during the reaction. This demonstrates that the reactions to $(\underline{\mathrm{A}})$ and $(\underline{\mathrm{B}})$ are competitive reactions.

\section{$3 \cdot 1$ Claisen Rearrangement of 2-allyloxy- benzoic Acid (AOBA)}

The rate constants of AOBA in three different solvents are shown in Table 1. The order of the reactivity is:

$$
\text { DEG }>\text { DEGMEE }>\text { decalin, }
$$

and appears to correspond to the polarity of these solvents. This phenomenon is normally recognized for the

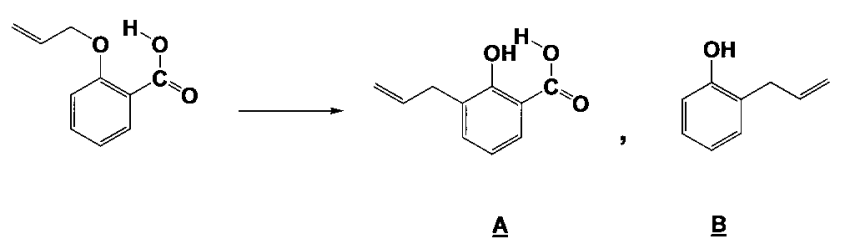

Fig. 1 Claisen Rearrangement of AOBA.

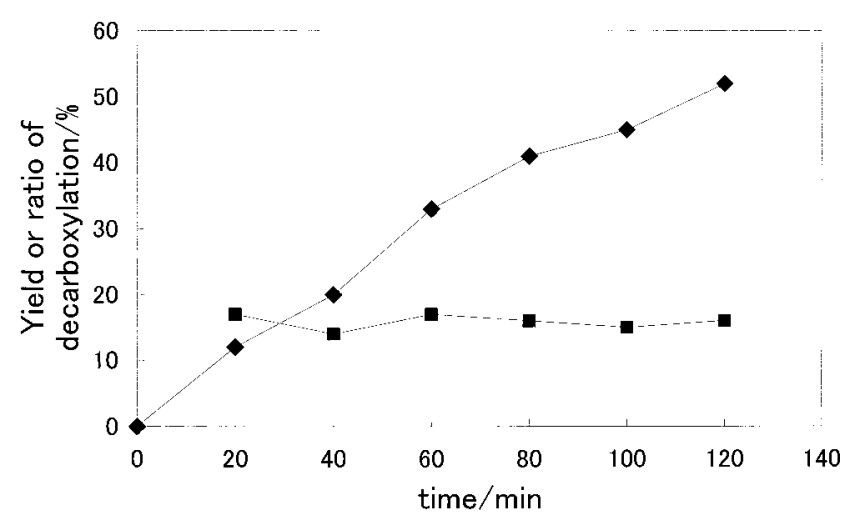

Fig. 2 Yield and Ratio of Decarboxylation of AOBA; $\diamond$ :Yield, $\mathbf{\square}$ :Ratio of decarboxylation.
Claisen rearrangement, and contributes to the solvation that stabilizes the transition state with a negative charge on the ethereal oxygen (3).

\section{$3 \cdot 2$ Claisen Rearrangement of the Alkali Metal Salts of AOBA}

The solvent effect for the alkali metal salts of AOBA was the same as that for the free AOBA. Namely, the reactivity in the polar solvent was higher than that in the nonpolar solvent (Table 1).

The order based on the type of metal was:

$$
\text { Li salt }>\mathrm{Na} \text { salt }>\mathrm{K} \text { salt. }
$$

This order is opposite to that of the ionic radius of the metal ions. In general, the degree of solvation of metal ions increases with the decreasing ionic radius. Therefore, the order suggests that the higher the degree of solvation, the higher the rearrangement reactivity. However, the reactivity of all these salts was lower than that of the free AOBA.

The authors reported an accelerated effect for the alkali metal salts of 2-allyloxyphenol (4). The accelerating effect for this substrate was due to the following factors. (a) Strong electron-releasing effect of $-^{-} \mathrm{O}^{-}$in metal oxide group. (b) Intramolecular repulsion between the oxygen of the ether and the $-\mathrm{O}^{-}$. For the alkali-metal salts of AOBA in this study, such an accelerated effect was not recognized. It can be attributed to the weakness of these two factors. For (a), the $-\mathrm{COO}^{-}$is electron-withdrawing which is different from the $-\mathrm{O}^{-}$. Concerning (b), the repulsion between the oxygen and the $-\mathrm{COO}^{-}$is a disadvantage compared to the $-\mathrm{O}^{-}$. Furthermore, the most important factor would be the loss of the intramolecular hydrogen bond (Fig. 3) between the carboxyl group and the ethereal oxygen that accelerates the rearrangement by decreasing the higher elec-

Table 1 Claisen Rearrangement Rate Constants of AOBA and its Metal Salts ${ }^{\mathrm{a}}$.

\begin{tabular}{lccc}
\hline & \multicolumn{3}{c}{$\mathrm{k}\left(\times 10^{5} \mathrm{sec}^{-1}\right)$} \\
\cline { 2 - 4 } & in decalin & in DEGMEE & in DEG \\
\hline AOBA & 3.0 & 5.2 & 7.6 \\
Li salt & 2.5 & - & 3.1 \\
Na salt & 0.6 & 0.9 & 1.6 \\
K salt & 0.6 & - & 1.5 \\
Ca salt & 16.7 & 6.4 & 2.2 \\
Ba salt & 11.1 & - & 1.1 \\
\hline
\end{tabular}

a) Reaction temperature is $140^{\circ} \mathrm{C}$. 


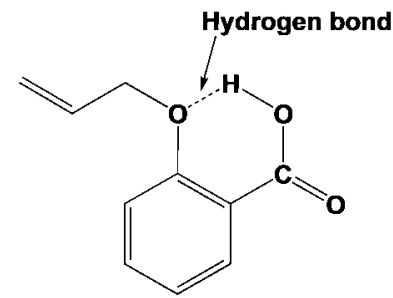

(a)

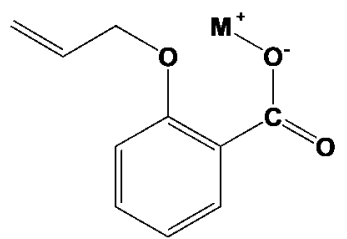

M: Li, Na, K

(b)
Fig. 3 Intramolecular Hydrogen Bond in Free AOBA (a) and No Interaction in its Alkali Metal Salt (b).

tron density on the ethereal oxygen in the transition state.

\subsection{Claisen Rearrangement of the Alka- line-Earth Metal Salts of AOBA}

The solvent effect for the alkaline-earth metal salts of AOBA was different from that for the free AOBA or its alkali metal salts. Namely, the reactivity in a nonpolar solvent was higher than that in a polar solvent (Table 1).

The complex-forming ability of alkaline-earth metal ions is much higher than that of the alkali metal ions. Therefore, in the alkaline-earth metal salts, a chelate ring would be formed between the ethereal oxygen and the $-\mathrm{COO}^{-}$(Fig. 4). This ring can be more stable in the transition state of the rearrangement because of an increase in the electron density of the ethereal oxygen. Similar catalysis mechanism was proposed for the rearrangement of allyl vinyl ethers with $\mathrm{Cu}$ and $\mathrm{Yb}$ salts (5). On the contrary, in a polar solvent, the reactivity was very low. It appears that the chelate effect is disturbed by the solvation of the alkaline-earth metal ions.

The order by the type of metals was:

$$
\text { Ca salt }>\text { Ba salt. }
$$

The $\mathrm{Ca}$ ion has a higher complex-forming ability than the $\mathrm{Ba}$ ion. The above-mentioned chelate effect would also be supported by this order.

As compared with AOBA and its alkali metal salts, the order of the reactivity in a polar solvent was:

free AOBA $>$ alkali metal salts

$$
=\text { alkaline-earth metal salts. }
$$

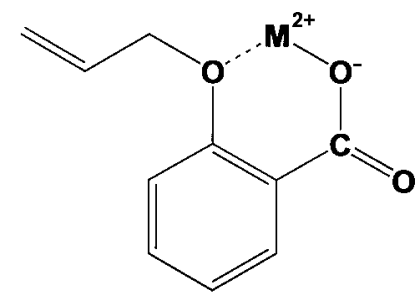

M: Ca, Ba

Fig. 4 Chelate Ring in Alkalineearth Metal Salt of AOBA.

In a nonpolar solvent, the order changed as follows: alkaline-earth metal salts $>$ free AOBA $>$ alkali metal salts.

Namely, no accelerated effect is recognized for metal salts in a polar solvent because the intramolecular hydrogen bond such as for the free AOBA is not able to form. Similarly, there is no accelerated effect for the alkali metal salts in a nonpolar solvent. However, a significantly high reactivity was observed for the alkalineearth metal salts in a nonpolar solvent. This is attributed to the chelate effect that is in excess of the intramolecular hydrogen bonding effect formed in free AOBA.

\section{References}

1. M. GOTO and T. HAYASHI, The Effect of the Substituents with Hydroxy Group on the Claisen Rearrangement of Allyl [o-(or $p$ )Substituted phenyl] Ethers, Nippon Kagaku Kaishi, 49-54 (1986).

2. D.S. TARBELL and T.W. WILSON, The Rearrangement of OCrotyl-3,5-dichlorosalicilic Acid and Related Compounds, $J$. Am. Chem. Soc., Vol. 64, 607-612 (1942).

3. M.J.S. DEWER and B.D. NAHLOVSKY, Claisen Rearrangement of Cinnamyl Phenyl Ether in Isotropic and Nematic Solvents and in a Clathrate, J. Am. Chem. Soc., Vol. 96, 460-465 (1974).

4. M. GOTO, T. HAYASHI, K. GODA and Y. OKADA, The Effect of Metal Oxido Group on the Claisen Rearrangement, Nippon Kagaku Kaishi, 1091-1101 (1986).

5. M. HIERSEMANN and L. ABRAHAM, The $\mathrm{Cu}(\mathrm{Otf})_{2}-$ and $\mathrm{Yb}(\mathrm{Otf})_{3}$-Catalyzed Claisen Rearrangement of 2-Alkoxycarbonyl-Substituted Allyl Vinyl Ethers, Org. Lett., Vol. 3, 49-52 (2001). 